\title{
Design of a Real-Time Adaptively Tuned Dynamic Vibration Absorber with a Variable Stiffness Property Using Magnetorheological Elastomer
}

\author{
Toshihiko Komatsuzaki and Yoshio Iwata \\ Institute of Science and Engineering, Kanazawa University, Kakuma-machi, Kanazawa, Ishikawa 920-1192, Japan \\ Correspondence should be addressed to Toshihiko Komatsuzaki; toshi@se.kanazawa-u.ac.jp
}

Received 21 October 2014; Accepted 10 December 2014

Academic Editor: Miao Yu

Copyright (C) 2015 T. Komatsuzaki and Y. Iwata. This is an open access article distributed under the Creative Commons Attribution License, which permits unrestricted use, distribution, and reproduction in any medium, provided the original work is properly cited.

An elastomer composite with controllable stiffness, known as a magnetorheological elastomer (MRE), is used in a dynamic vibration absorber whose natural frequency is tuned adaptively to the disturbance frequency through the application of an external magnetic field. The field-dependent property test of the fabricated MRE sample shows that the stiffness changes by more than six times compared to the baseline property value at a $40 \%$ iron powder volume concentration. The MRE is then used to fabricate a frequencytunable dynamic absorber for mitigating transient vibrations of a one-degree-of-freedom system. Investigations show that the proposed absorber outperforms a conventional passive-type absorber throughout the tunable frequency range.

\section{Introduction}

A passive-type dynamic vibration absorber (DVA) is basically a mass-spring system that suppresses the vibration of a structure at a particular frequency. Since the natural frequency of the DVA is usually tuned to a frequency of particular excitation, the DVA is especially effective when the excitation frequency is close to the natural frequency of the structure. Fixing the physical properties of the DVA limits the application to a narrowband, harmonically excited vibration problem. The design of the absorber, adhering to a well-known optimal tuning and damping theory, can extend the effective frequency range [1]. However, the damping performance remains at a certain level irrespective of whether the vibration is harmonic or not, and the performance may become worse for vibrations caused by transient disturbances. A frequency-tunable DVA that can modulate its stiffness provides adaptability to the vibration control device against nonstationary disturbances. Several studies have been reported in this regard [2-5] but the implementation of such adaptability would be complex and the response time may become a design issue.
The magnetorheological elastomer (MRE) belongs to the class of smart materials whose elastic property can be varied according to an externally applied magnetic field. The robustness and structural simplicity, stiffness variability, and fast response properties of MREs hold promise for the new design of semiactive adaptive vibration control devices, following numerous publications of a number of studies on this topic [6-16].

Komatsuzaki et al. [6] and Liao et al. [7] have developed an MRE-based vibration isolator where real-time semiactive vibration control techniques are applied in order to reduce vibration in the structure or the payload. Previously published studies also include development of the adaptive absorbers using MREs. Deng and Gong [8] have proposed a tunable dynamic absorber using MRE where a natural frequency shift of $155 \%$ could be obtained when a magnetic field of $1 \mathrm{~T}$ was applied that consequently damped the beam vibration effectively. Lerner and Cunefare [9] have studied MRE-based vibration absorbers in which MREs are deformed under three different configurations. They have found the configuration and the iron concentration of MREs that maximize the natural frequency shift of the absorber. 
While extending the frequency shift property of the absorber, possible influences on the damper performance of the ratio of the primary and the adjacent masses, the tunable range modulations, and the damping property of the material itself have not yet been elucidated. Furthermore, these prior studies have been completed under a harmonic disturbance condition that is less likely to be observed in real applications such as the transient vibration in vehicles. On this issue, Hoang et al. [10] have analytically investigated the real-time control of transient vibration in vehicular power trains using an MRE-based, adaptively tuned dynamic absorber; however, the implementation of such a scheme to the actual equipment has not been realized thus far.

In this paper, an effective design of the adaptively tuned dynamic absorber is shown against the target structure in order to maximize the performance of the absorber with a frequency-tunable feature. The performance of the proposed MRE-based DVA is evaluated by comparison to a passivetype absorber with fixed properties. Additionally, the study aims to show numerically as well as experimentally that the real-time adaptive control is quite possible for a transient vibration caused by excitation, whose frequency changes with time. Field-dependent properties of the fabricated MREs are first shown. The MREs are then introduced into a DVA whose frequency adjustability is evaluated. Finally, the real-time vibration control performance of the frequencytunable absorber for a base-excited, one-degree-of-freedom system is evaluated. Investigations show that the vibration of the structure can be effectively reduced with an improved performance by the DVA in comparison to the conventional passive-type absorber.

\section{Description of Magnetorheological Elastomer}

A magnetorheological fluid (MRF) is a well-known kind of functional material, where the ferromagnetic particleswhose size is usually distributed between 1 and $10 \mu \mathrm{m}$-are homogeneously dispersed within the carrier fluid, in the absence of an external magnetic field. Under the presence of a magnetic field, magnetically polarized particles form chainlike structures according to the attractive forces amongst each other that produce changes in the apparent viscosity of the fluid. A number of engineering applications are investigated regarding the fluid owing to its highly variable and responsive properties [17, 18]. However, the fluid causes several problems, such as sealing, sedimentation, or aggregation of particles.

The elastomer composites incorporating ferrous particles, known as MREs, have been developed with the aim of overcoming the problems associated with the fluid. Fixation of iron particles within the elastomeric matrix provides MREs with acquired advantages when installed as mechanical elements, in which the sedimentation problems can be avoided and the sealing problems can be neglected. Moreover, their shape is sustained permanently and it is easily molded to fit various configurations. On the other hand, the particles are strongly immobilized and therefore the characteristic variation range in MREs usually becomes smaller compared to MRFs. It should also be noted that the MRE is characterized by an elastic property rather than a viscous property and hence a viscoelastic evaluation is necessary.

Several studies have been reported regarding MREs [616]. Prior research work includes a fundamental investigation of the material in order to improve the property variation range of MREs. For example, Gong et al. [12] have studied isotropic MREs where the silicone oil was introduced in the mixture of silicone rubber and iron particles to improve mobility of particles within the elastomeric material, and their shear storage modulus was evaluated by changing their compositions. Zhang et al. [13] have proposed the fabrication process to form the patterned structure within MREs and have investigated the structural effect on their stress-strain relationship. Others have numerically predicted the mechanical response of MREs in the presence of a magnetic field, as described in [14-16]. It seems that a welldefined fabrication process, a reliable evaluation, and the analytical models of MREs have not yet been determined. However, MREs are obviously the prospective materials for new engineering applications especially in the field of mechanical and structural vibrations.

2.1. Fabrication of MRE. The fabricated MRE samples are composed of carbonyl iron powder of an approximate diameter of $10 \mu \mathrm{m}$ dispersed within the room-temperaturevulcanizing (RTV) rubber matrix. They are fabricated based on the expertise acquired in earlier studies $[12,13]$. The stiffness of the elastomeric matrix should be kept low to ensure a range in property variation when exposed to an external magnetic field. Additionally, the application of the magnetic field during the curing process of MRE is known to be effective for expanding the shear modulus variation. Elastomers are formed in square cuboids with a size of $25 \mathrm{~mm}$ and a thickness of $10 \mathrm{~mm}$ and with different iron volume contents ranging from 30 to $50 \%$.

2.2. Dynamic Property Test. The viscoelastic properties of the fabricated MREs are investigated by measuring their dynamic response using the experimental setup shown in Figure 1. The upper part of the electromagnet (whose mass is $1.56 \mathrm{~kg}$ ) and two MREs placed between the upper and the lower parts of the electromagnet are assumed to emulate a mass and two springs, respectively, and they constitute a onedegree-of-freedom (DOF) vibration system. While exciting the system horizontally by an exciter, the base and mass accelerations are measured and processed in the form of a transfer function by an FFT analyzer. The frequency response of the system is measured through a random excitation at the cutoff frequency of $100 \mathrm{~Hz}$, while the applied electric current is varied from 0 to $4 \mathrm{~A}$.

Based on the measured acceleration transfer function, the complex spring constant is calculated according to the 


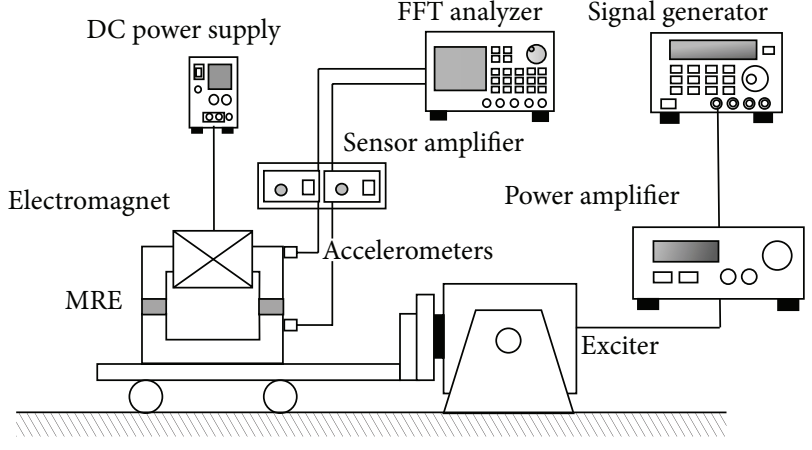

(a)

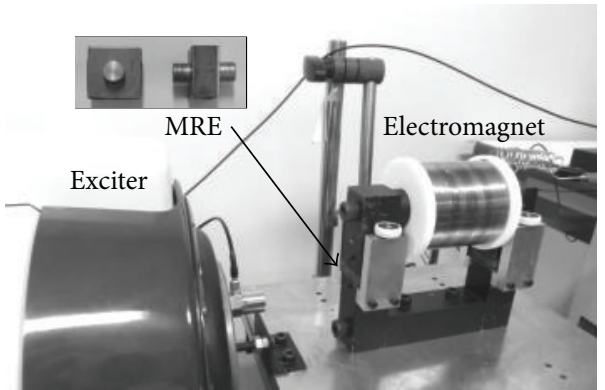

(b)

FIGURE 1: Viscoelastic property measurement system: (a) schematic and (b) photograph.

TABLE 1: The identified spring constants of MREs listed against applied current. All values are represented in units of N/mm. Numbers shown in parentheses are the elastic shear moduli in MPa.

\begin{tabular}{|c|c|c|c|c|c|}
\hline \multirow{2}{*}{ MRE composition } & \multicolumn{5}{|c|}{ Current } \\
\hline & $0 \mathrm{~A}$ & $1 \mathrm{~A}$ & $2 \mathrm{~A}$ & $3 \mathrm{~A}$ & $4 \mathrm{~A}$ \\
\hline 30 vol\% MRE & $17.1(0.27)$ & $27.3(0.44)$ & $40.3(0.65)$ & $54.3(0.87)$ & $62.4(1.00)$ \\
\hline 40 vol\% MRE & $28.5(0.46)$ & $53.9(0.86)$ & $91.8(1.47)$ & $135(2.15)$ & $157(2.51)$ \\
\hline 50 vol\% MRE & $62.2(1.00)$ & $98.2(1.57)$ & $163(2.61)$ & $254(4.06)$ & $301(4.81)$ \\
\hline
\end{tabular}

following equation. The equation of motion of the one-DOF system is thus written as

$$
m \ddot{x}=-k^{*}(x-u),
$$

where the symbols $x, u, m$, and $k^{*}$ represent the displacement of the system, the displacement of the shaking table, the mass, and the complex spring constant, respectively. Introduction of the relative displacement, $y$, between the mass and the shaking table leads to a modified form of (1) as follows:

$$
m \ddot{y}+k^{*} y=-m \ddot{u} .
$$

The frequency-dependent complex spring constant, $k^{*}$, is defined in accordance with the following manner:

$$
k^{*}(\omega)=k_{0} \cdot v(\omega)\{1+j \eta(\omega)\} .
$$

In (3), $k_{0}$ signifies the reference spring constant calculated by the natural frequency $\omega_{0}$ of the system when no magnetic field is applied, $v(\omega)$ is the frequency-dependent normalized spring constant, and $\eta(\omega)$ is the loss factor. From (2) and (3), the acceleration transfer function is derived according to the following equation [19]:

$$
G(j \omega)=\frac{v(\omega)+j \eta(\omega) v(\omega)}{\left\{v(\omega)-\lambda^{2}\right\}+j \eta(\omega) v(\omega)}
$$

In (4), $\lambda=\omega / \omega_{0}$. By separating the real and the imaginary parts of the transfer function as $G(j \omega)=G_{R}+j G_{I}, v(\omega)$ and $\eta(\omega)$ can be expressed using $G_{R}$ and $G_{I}$, as

$$
\begin{aligned}
& v(\omega)=\frac{G_{R}\left(G_{R}-1\right)+G_{I}^{2}}{\left(G_{R}-1\right)^{2}+G_{I}^{2}} \lambda^{2}, \\
& \eta(\omega)=\sqrt{-1+\frac{\left(2 G_{R}-1\right) \lambda^{2}}{\left(G_{R}-1\right) v(\omega)}-\frac{G_{R} \lambda^{4}}{\left(G_{R}-1\right) v(\omega)^{2}}} .
\end{aligned}
$$

Figure 2 shows the viscoelastic property of MREs at different iron concentrations. The normalized stiffness and the loss factor are calculated using (5) for each sample. Both properties are almost independent of frequency in all cases. In this study, the nominal value of the spring constant in the absence of a magnetic field is $15.9 \mathrm{~N} / \mathrm{mm}$ for an MRE of $30 \%$ iron volume content (vol\%), $24.6 \mathrm{~N} / \mathrm{mm}$ for that with a $40 \mathrm{vol} \%$, and $58.2 \mathrm{~N} / \mathrm{mm}$ for that with a $50 \mathrm{vol} \%$. Based on the nominal value, the identified stiffness values for each MRE and for various applied currents are listed in Table 1. Indicated values are averaged over the frequency range of 20 to $80 \mathrm{~Hz}$. The normalized stiffness has the lowest value when no magnetic field is applied, and it becomes larger in value according to the strength of the field owing to the increase in interparticle forces within the elastomeric matrix. On the other hand, the change of the loss factor seems to be small enough in comparison to the change of the stiffness, irrespective of the presence of the magnetic field, whose value becomes approximately 0.4 in all cases. Such a high damping value is attributed to the viscous property of the host elastomer. The same holds true for the average 

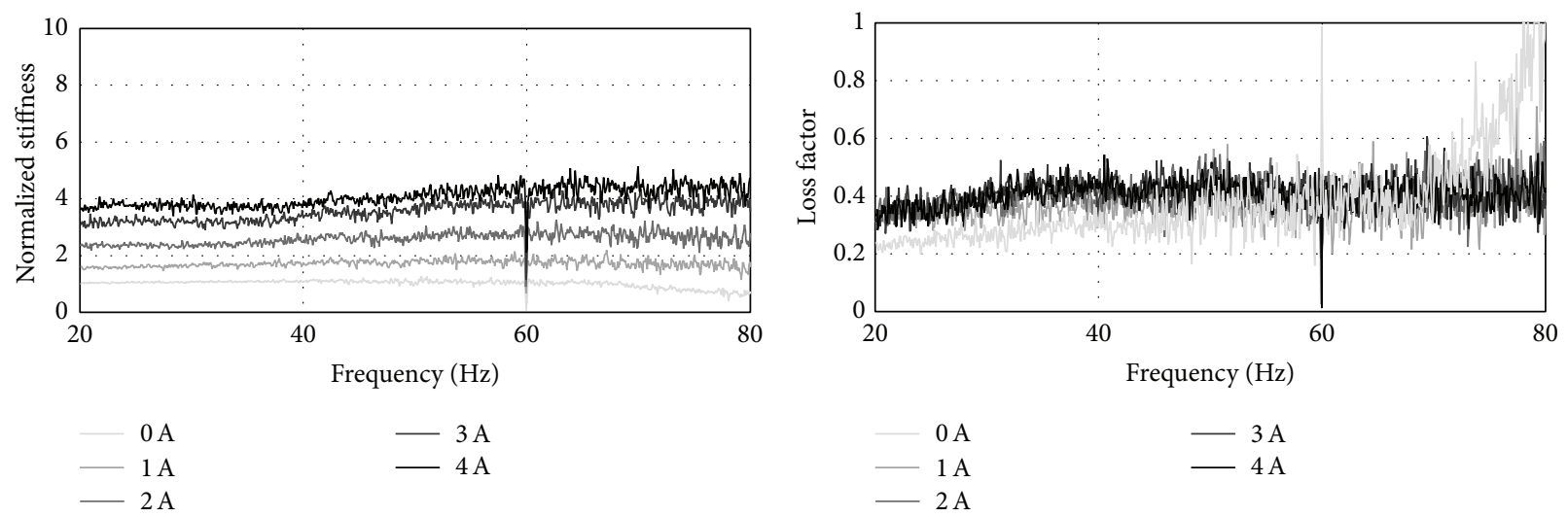

(a)
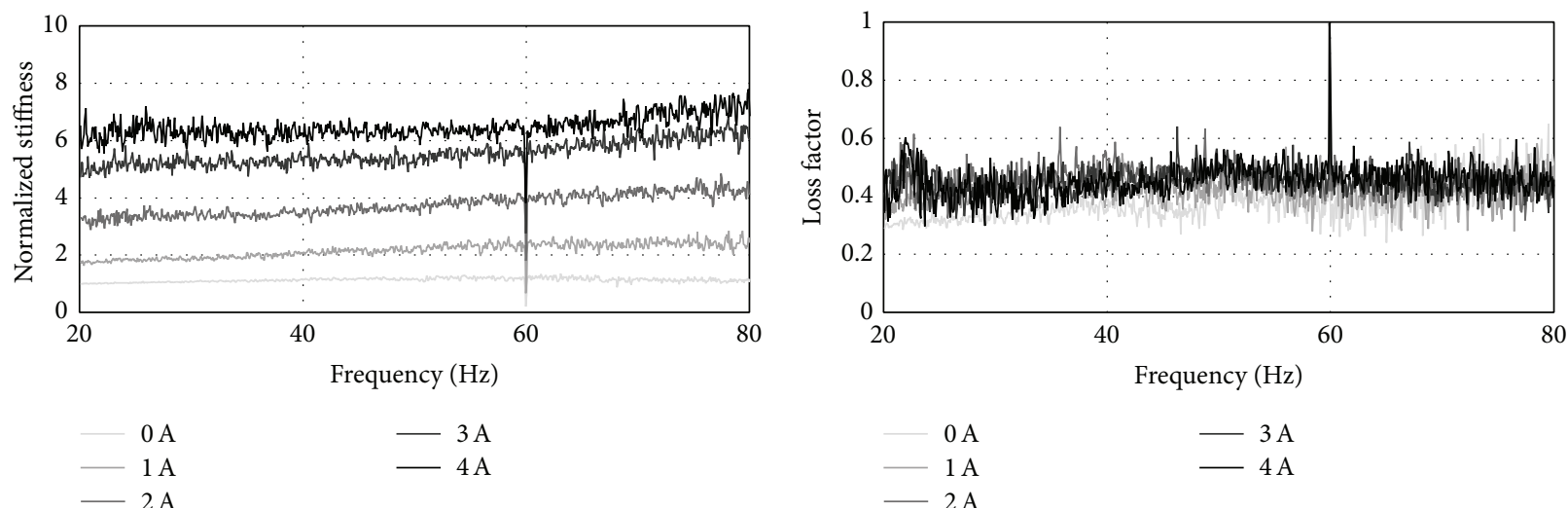

(b)
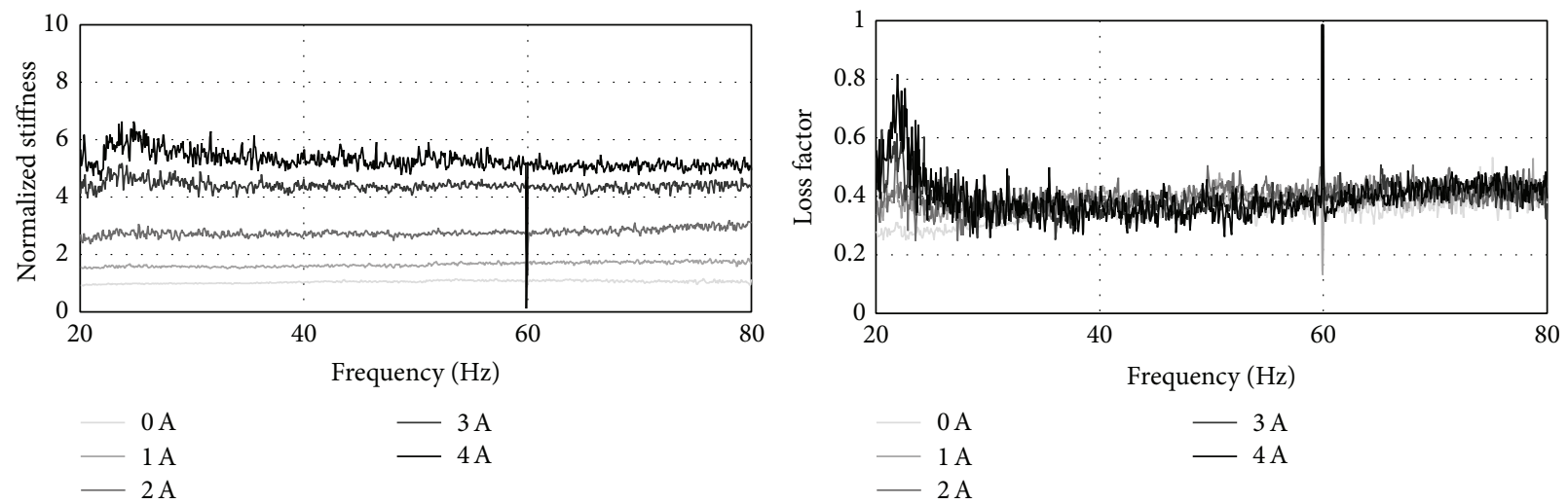

(c)

Figure 2: Complex stiffness constant of magnetorheological elastomer (MRE) samples: (a) MRE of 30 vol\% iron content, (b) 40 vol\%, and (c) $50 \mathrm{vol} \%$. The left figures in the left column show the normalized spring constant and the figures in the right column show the loss factor.

stiffness magnification and the loss factor shown in Figure 3. Additionally, the maximum stiffness variation is observed for the MRE sample with 40 vol\% iron content. The higher volume content of iron particles leads to the expression of the stronger interparticle forces, whereas the base hardness of the elastomer itself also becomes high. Hence, the largest magnification of stiffness is provided by a certain optimum composition between the elastomeric matrix and the iron particles.

\section{Numerical Prediction of Performance of the Dynamic Absorber}

In this section, the damping performance of a dynamic absorber with a variable stiffness is numerically predicted for a harmonically excited response of a two-DOF vibration system incorporating the absorber, where the tuning capacity is based on the measured property of the prototypes. 


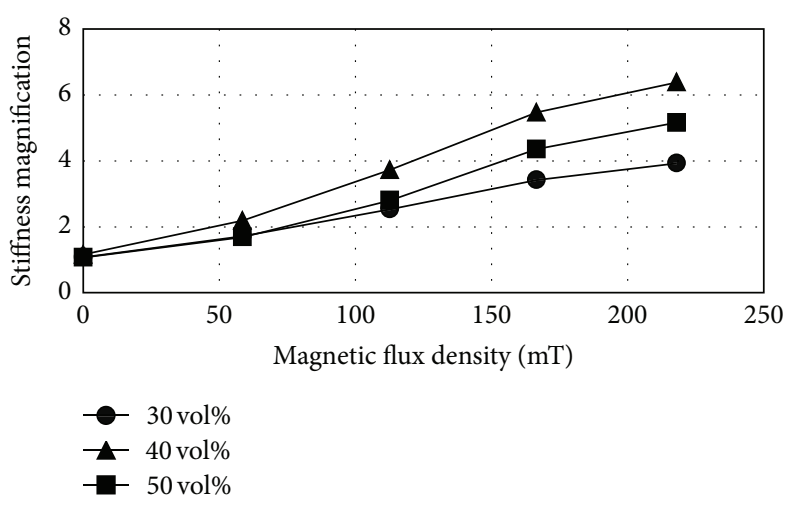

(a)

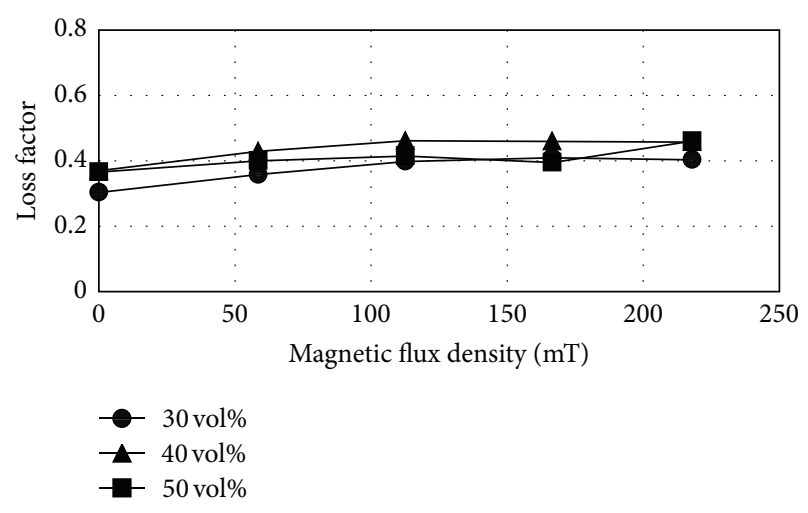

(b)

FIGURE 3: Average stiffness magnification and loss factor of magnetorheological elastomers (MREs) plotted against the magnetic flux density: (a) the stiffness variation and (b) the loss factor variation.

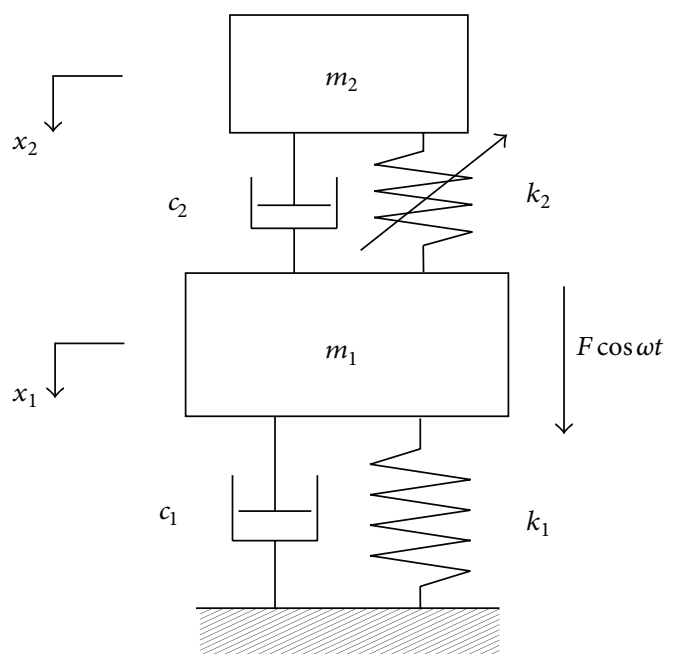

FIgURE 4: Analytical model of dynamic absorber with variable stiffness attached to a primary system.

3.1. Numerical Model of Two-DOF System Including a Variable Stiffness Absorber. A mathematical model of a twoDOF vibration system consisted of a primary system and a secondary system, and having a variable stiffness property, as shown in Figure 4 . The primary system, having a mass of $m_{1}$, a spring constant, $k_{1}$, and a damping coefficient, $c_{1}$, is excited harmonically by the external force $F \cos \omega t$, whereas the stiffness variability is assigned to the spring element, $k_{2}$, of the adjacent system, whose property changes according to the actual measured characteristic. Assuming that the stiffness $k_{2}$ is constant, the equations of motion for the two-DOF system are written as follows, using symbols shown in Figure 4:

$$
\begin{gathered}
m_{1} \ddot{x}_{1}+\left(c_{1}+c_{2}\right) \dot{x}_{1}+\left(k_{1}+k_{2}\right) x_{1} \\
-c_{2} \dot{x}_{2}-k_{2} x_{2}=F \cos \omega t, \\
m_{2} \ddot{x}_{2}+c_{2} \dot{x}_{2}+k_{2} x_{2}-c_{2} \dot{x}_{1}-k_{2} x_{1}=0 .
\end{gathered}
$$

Equation (6) is further transformed into a nondimensional form with respect to time and displacement, in order to illustrate the effect of the variable frequency range on the damping performance in a qualitative manner. By dividing the real displacements and time by the characteristic length $x_{s t}=F / k_{1}$ and the characteristic time $T_{c}=\sqrt{m_{1} / k_{1}}$, a nondimensional form of (6) is derived as follows:

$$
\begin{array}{r}
\ddot{p}+2\left(\zeta_{1}+\mu z \zeta_{2}\right) \dot{p}+\left(1+\mu z^{2}\right) p \\
-2 \mu z \zeta_{2} \dot{q}-\mu z^{2} q=\cos \Omega \tau, \\
\ddot{q}+2 z \zeta_{2}(\dot{q}-\dot{p})+z^{2}(q-p)=0 .
\end{array}
$$

The parameters in (7) are defined as $\Omega=\omega / \omega_{1}, z=\omega_{2} / \omega_{1}$, $p=x_{1} / x_{s t}, q=x_{2} / x_{s t}, \omega_{1}^{2}=k_{1} / m_{1}, \omega_{2}^{2}=k_{2} / m_{2}, \mu=m_{2} / m_{1}$, $\zeta_{1}=c_{1} / 2 \sqrt{m_{1} k_{1}}$, and $\zeta_{2}=c_{2} / 2 \sqrt{m_{2} k_{2}}$.

\subsection{Optimal Design of the Damped Dynamic Vibration} Absorber. The optimal tuning and damping theory is well known for the design of the fundamental mass-linear spring type, damped dynamic absorber [1]. In the subsequent section, the performance of the variable tuned absorber is compared with the performance of the optimally tuned absorber with fixed properties. Defining the mass ratio between the primary and the adjacent systems as $\mu$, the conclusive form of the optimally tuned condition is expressed as follows:

$$
\frac{\omega_{2}}{\omega_{1}}=\frac{1}{1+\mu}
$$

Additionally, the optimal damping ratio, $\zeta_{\mathrm{opt}}$, with respect to the damped vibration absorber is determined by (9), where the maximum amplitude of the primary system response curve is found at either location of two fixed points:

$$
\zeta_{\mathrm{opt}}=\sqrt{\frac{3 \mu}{8(1+\mu)^{3}}} .
$$




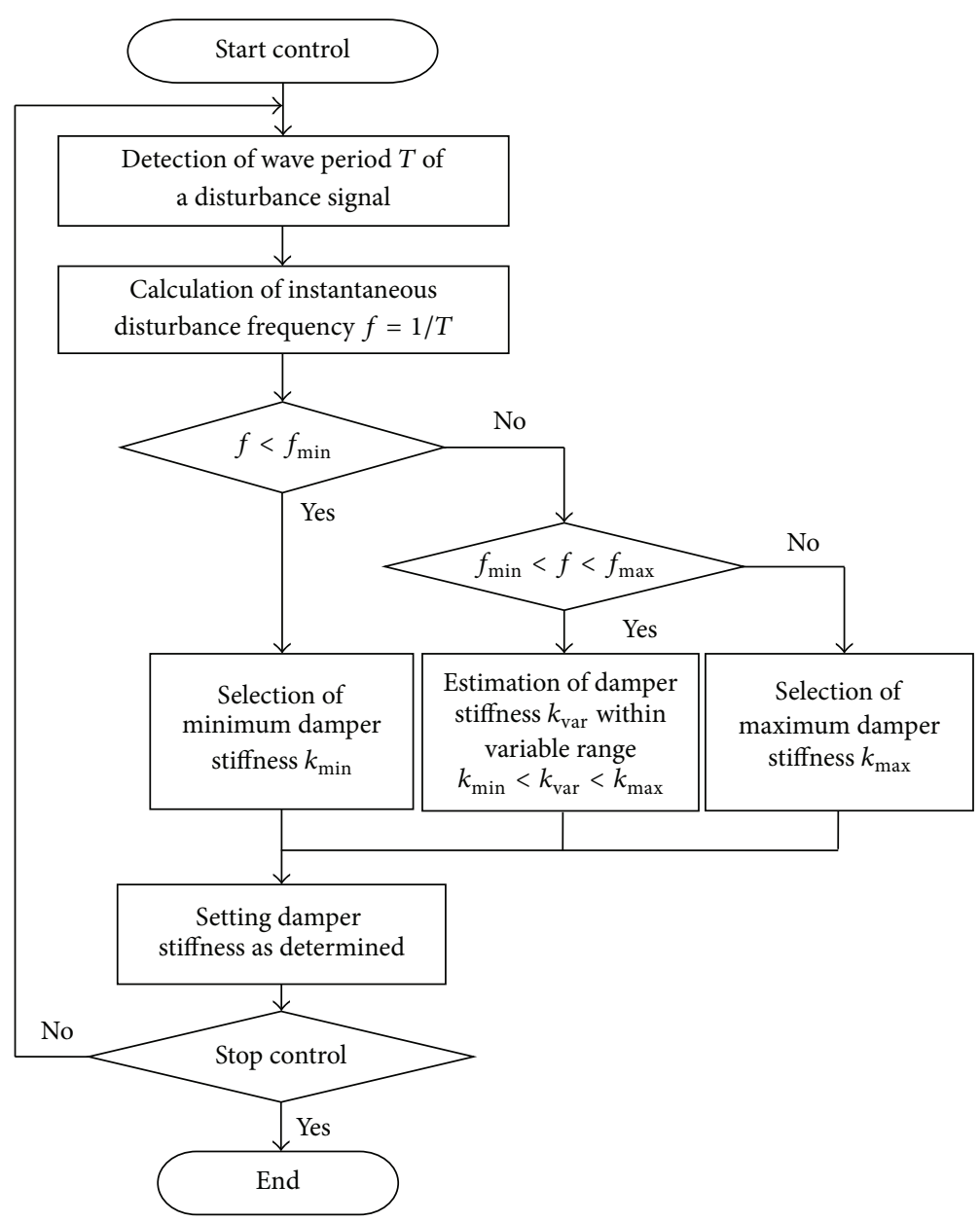

FIgURE 5: Flow diagram of the stiffness switching rules for the tuned dynamic absorber.

3.3. Calculated Response of Two-DOF System Incorporating Variable Stiffness Absorber. Based on the nondimensional (7) and on the measured frequency variation range of the absorber (that will be described later), the damping performance of the absorber with variable stiffness is numerically predicted. In the calculation, three different mass ratios $\mu=$ $0.1,0.2$, and 0.3 are configured. The variable frequency range of the absorber for each mass ratio is shown in Table 2 in conjunction with the optimal design parameters of the case with fixed property. Considering that the actual frequencytunable range extended to 1.4 times the baseline value, the natural frequency of a one-DOF system is located at the middle of the range when the absorber is fixed rigidly to the primary system. As shown in Figure 5, the natural frequency of the adjacent system is tuned adaptively to match the frequency of a harmonic external force within the variable range, whereas the damper property is fixed at the minimum or the maximum stiffness value for the lower and upper outer bounds.

Figure 6 shows the primary system response for the case of a mass ratio $\mu=0.2$, when the external harmonic force is applied to the primary system as an input. The response amplitudes are expressed in nondimensional form using the static deflection $\delta_{0}=F / k_{1}$. In this figure, the optimally tuned damper response is also shown. It is shown from the figure that the overall response for the variable stiffness case can be minimized. Each tuned frequency of the response curve approximately corresponds to the frequency of the antinode of the primary system response at the respective damper stiffness variations, within the tunable frequency range. On the other hand, in the case of an optimally tuned absorber, the amplitude of the response curve remains at the level of the amplitude of the two fixed points within the target frequency range.

The responses of the variable stiffness absorbers at three different mass ratios are further compared in Figure 7. Even though the overall response can be improved, a distinct peak will appear for the case of a mass ratio of 0.1. If the mass of the adjacent system is relatively small while the damping ratio is kept constant, the response at the antinode cannot be fully reduced. In contrast, the primary system can be damped more effectively by increasing the damper mass. The response can be improved by reducing the damping ratio of the absorber by combining the MRE with other lightly damped high-stiffness elements, such as metals, or by setting the appropriate mass ratio in the vicinity of 0.2 . 


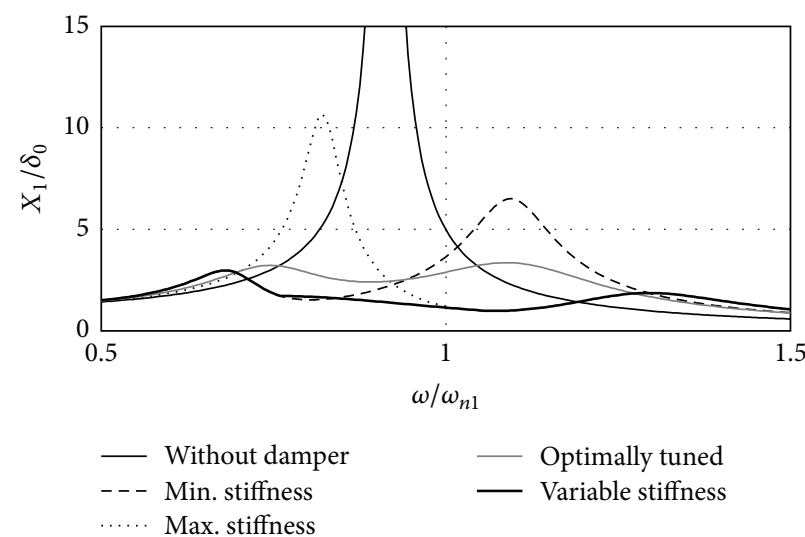

(a)

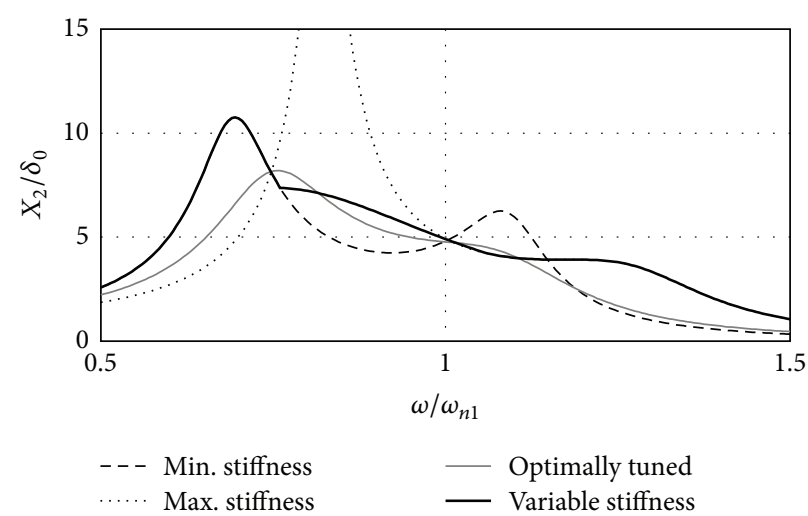

(b)

FIGURE 6: Calculated frequency response of a two-degree-of-freedom (DOF) system under harmonic excitation: (a) the primary system and (b) the stiffness-variable dynamic vibration absorber.

TABLE 2: System parameter used in numerical calculation.

\begin{tabular}{lccc}
\hline Mass ratio (absorber/primary) & 0.1 & 0.2 & 0.3 \\
Tuned frequency ratio (absorber/primary) & 0.91 & 0.83 & 0.77 \\
Optimal damping ratio (for absorber) & 0.17 & 0.21 & 0.23 \\
Frequency ratio (when absorber is immobilized) & 0.95 & 0.91 & 0.88 \\
Variable frequency range of absorber & $0.79-1.11$ & $0.76-1.06$ & 0.008 \\
Damping ratio (primary system) & & 0.12 \\
Damping ratio (absorber) & & 0.1 .03 \\
\hline
\end{tabular}

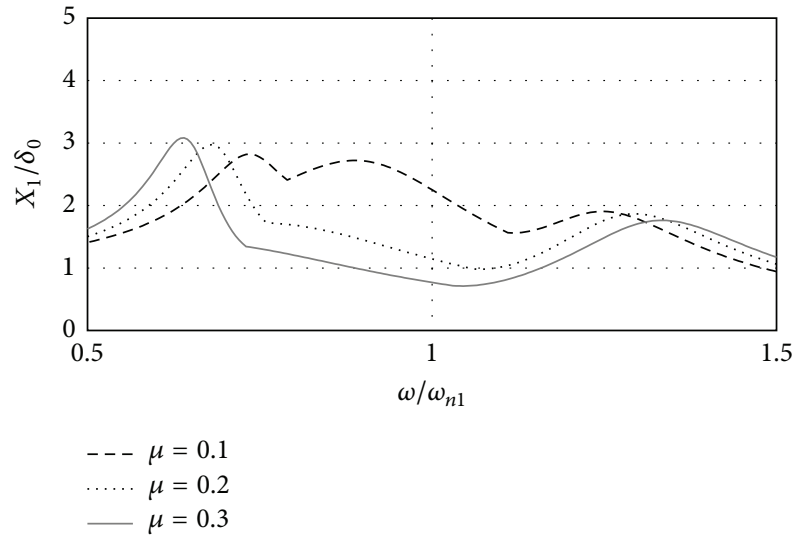

FIGURE 7: Frequency responses of a primary system compared by mass ratio between the primary and the secondary masses.

\section{Development of a Dynamic Absorber with Variable Stiffness Using MRE}

4.1. Fundamental Property of the Dynamic Absorber. A frequency-tunable DVA is further developed using the aforementioned MRE. A photograph of the dynamic absorber incorporating the MRE as a variable stiffness element is shown in Figure 8. The outer frame is made of steel and constitutes a closed magnetic path. A magnetic coil located at the center works as the mass $(370 \mathrm{~g})$ of the absorber that also generates the magnetic flux. Two MREs with thicknesses of $10 \mathrm{~mm}$ and diameters of $20 \mathrm{~mm}$ are mounted on the upper and lower parts of the coil so that the stiffness will change owing to the magnetic flux penetration. A $1 \mathrm{~mm}$ diameter wire is used to wind the coil with five hundred and forty turns.

The ability of the proposed absorber to change both natural frequency and damping ratio against the applied magnetic field was evaluated. The impulse response of the absorber was measured in the form of a time history at each applied current varying from 0 to $4 \mathrm{~A}$, and the natural frequency and the damping ratio are then calculated.

The measurement results of the fundamental properties of the dynamic absorber with variable stiffness are summarized in Figure 9 and Table 3, for each type of absorber with MREs of different iron concentrations. In the case of MRE with $40 \mathrm{vol} \%$ iron content, the largest natural frequency magnification ratio is observed. In this case, the baseline frequency of $25.8 \mathrm{~Hz}$ in the absence of a magnetic field is extended by a factor of 1.4 when a $4 \mathrm{~A}$ current is applied. In addition to the fact that the induced magnetic flux density at the same level of the applied current is about half of the density induced in the dynamic property test apparatus, all of the tightness of joints among elastomers, the core frame, and the electric coil affect the resulting frequency variation characteristics. On the other hand, the observed damping ratio is thought to have a constant value of 0.12 on average.

4.2. Investigation of the Damping Performance of the RealTime Tuned Dynamic Absorber. Based on the numerical prediction and the measured results of the fundamental 
TABLE 3: Tunable frequency range of the dynamic absorber with variable stiffness.

\begin{tabular}{lcccc}
\hline Iron concentration & Frequency variation range $[\mathrm{Hz}]$ & Bandwidth $[\mathrm{Hz}]$ & Magnification to the baseline & Damping ratio \\
\hline 30 vol\% & $21.1-29.2$ & 8.1 & 1.38 & 0.13 \\
40 vol\% & $25.8-36.0$ & 10.2 & 1.39 & 0.12 \\
50 vol\% & $36.6-46.8$ & 10.2 & 1.28 & 0.18 \\
\hline
\end{tabular}
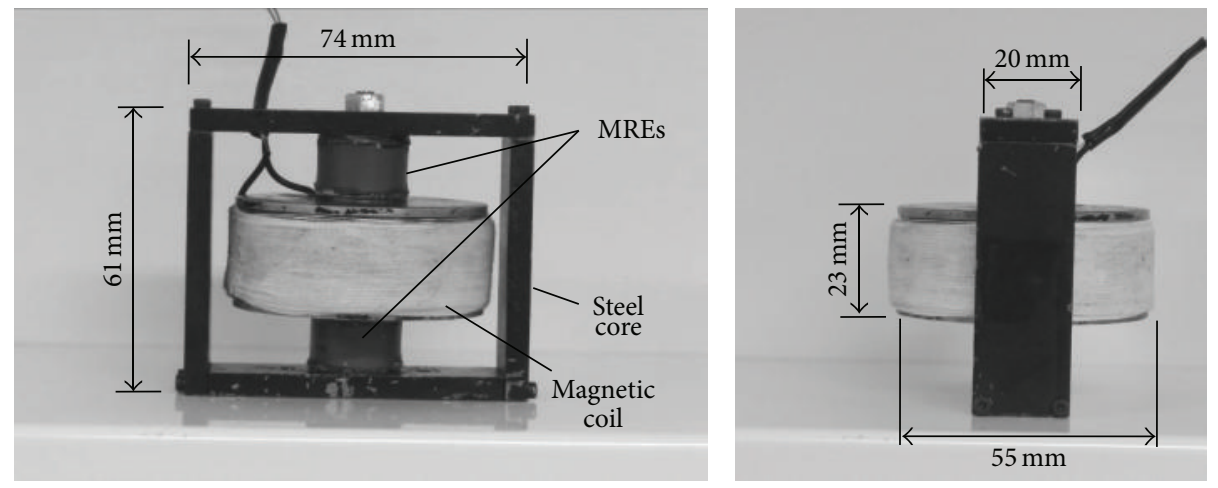

FIGURE 8: Schematic of the dynamic absorber with variable stiffness.

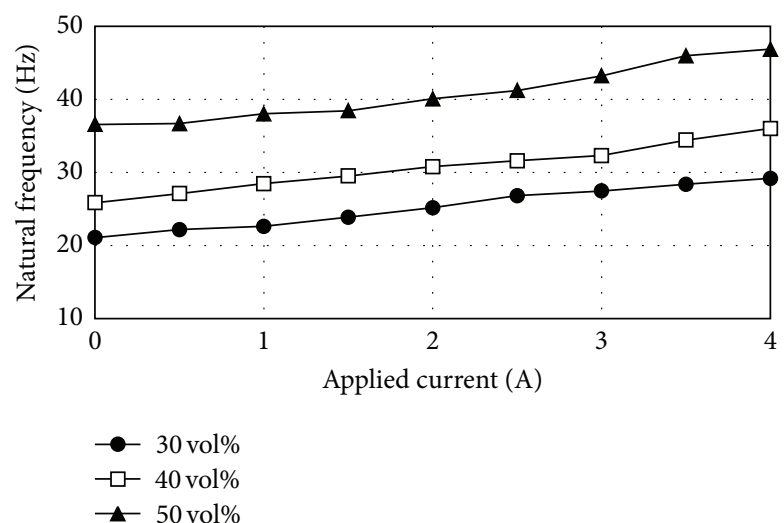

(a)

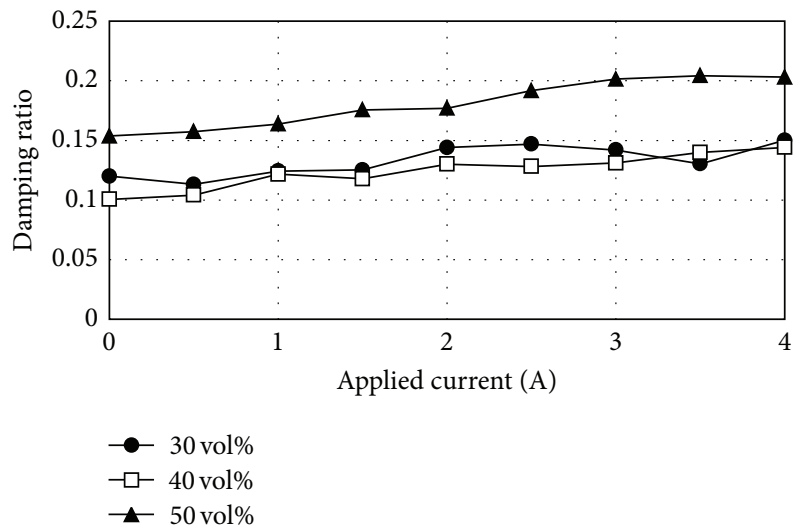

(b)

FIGURE 9: Fundamental property of the dynamic absorber with variable stiffness: (a) natural frequency variation and (b) damping ratio variation.

property of the absorber with variable stiffness, the damping performance is experimentally evaluated for the vibration control of a one-DOF system, as shown in Figure 10. The absorber incorporating an MRE with 40 vol\% iron content is adopted. The ratio between the primary and the damper masses is chosen to be 0.25 . The damping ratio of the primary system alone is also found to be 0.01 . Since it is difficult to assign the optimal damping property to the absorber for the present configuration, the optimally damped case is not considered in the experiment. The transient response of the system is measured when the entire structure is excited by a swept-sine input at the shaking table, whose frequency varies from 10 to $50 \mathrm{~Hz}$ at the speed of $2 \mathrm{~Hz} / \mathrm{s}$. The block diagram of the real-time control scheme is shown in Figure 11. The same real-time stiffness switching rule is applied as in the case of the numerical model.
The frequency responses obtained by the experiment are shown in Figure 12. The curve with a single resonant peak in Figure 12(a) corresponds to the case where the absorber mass is rigidly fixed to the primary structure, whereas the other solid lines correspond to responses when the damper stiffness is fixed at the respective applied currents. The remaining curve with dashed lines represents the response when the natural frequency of the absorber is adaptively tuned to the instantaneous frequency of the disturbance. The figure shows that the tunable absorber sustainably reduces the primary system response amplitude to as small as the minimum amplitude, within the variable range of 25 to $35 \mathrm{~Hz}$. The same can be said for the time histories shown in Figure 13, where an increase of amplitude near the resonance is constantly suppressed by the tuned absorber. In Figure 12, the optimally tuned case approximately corresponds to the response curve 


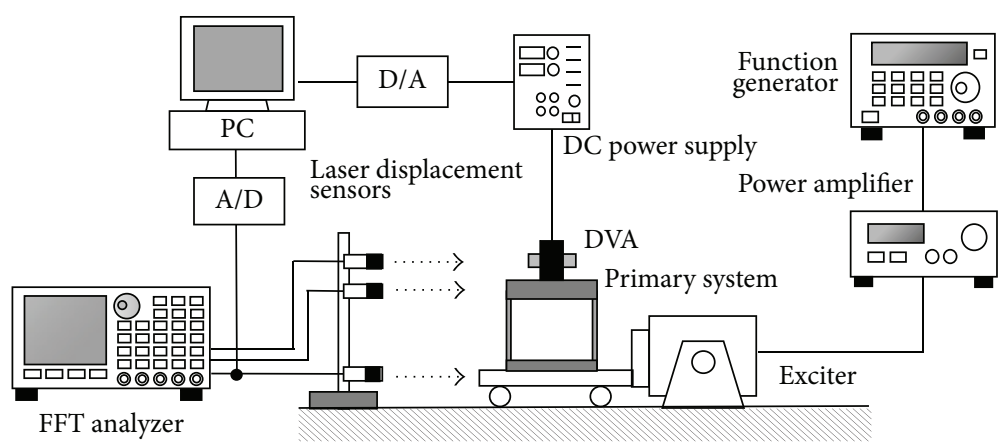

(a)

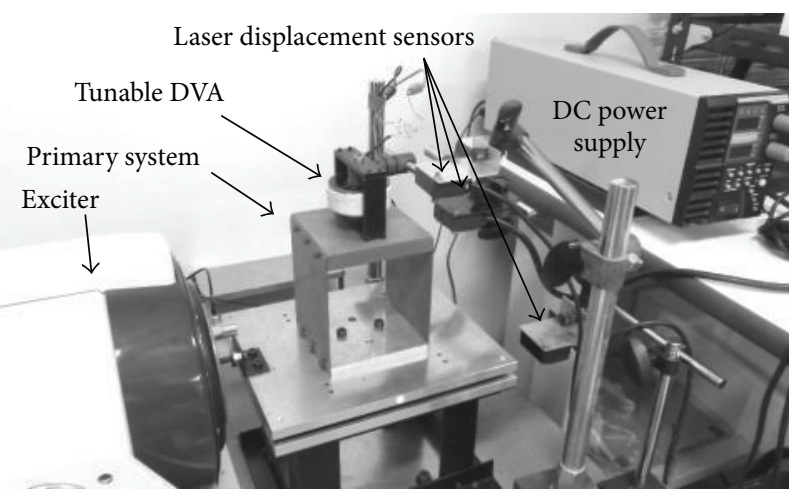

(b)

FigURE 10: Experimental setup of base-excited primary system damped by stiffness-variable DVA: (a) schematic and (b) photograph.

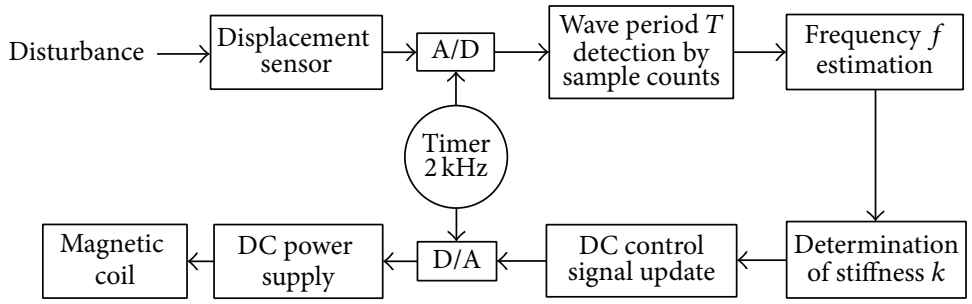

FIGURE 11: Block diagram of the controller for real-time frequency tuning of the absorber.
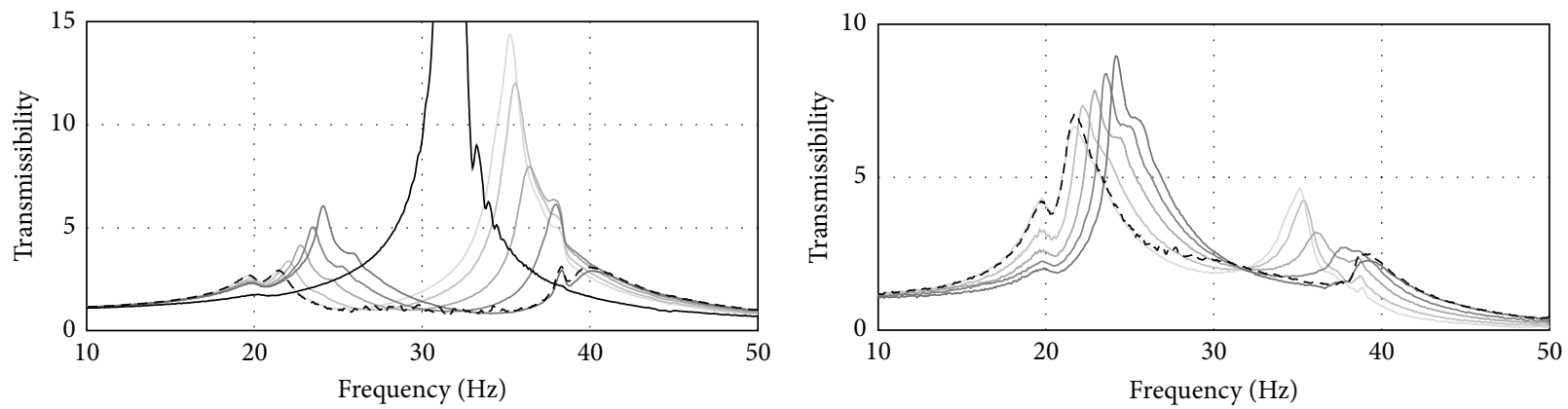

$$
\begin{array}{r}
0 \mathrm{~A} \\
-\quad 1 \mathrm{~A} \\
-\quad 2 \mathrm{~A} \\
-\quad 3 \mathrm{~A}
\end{array}
$$

$$
\begin{aligned}
& -4 \mathrm{~A} \\
& --- \text { Variable } \\
& - \text { Without damper }
\end{aligned}
$$

(a)

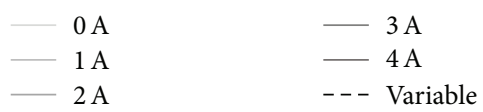

(b)

FIGURE 12: Response curves when the tunable DVA with MRE composed of $40 \%$ iron concentration is applied: (a) the primary system response and (b) the damper response. 


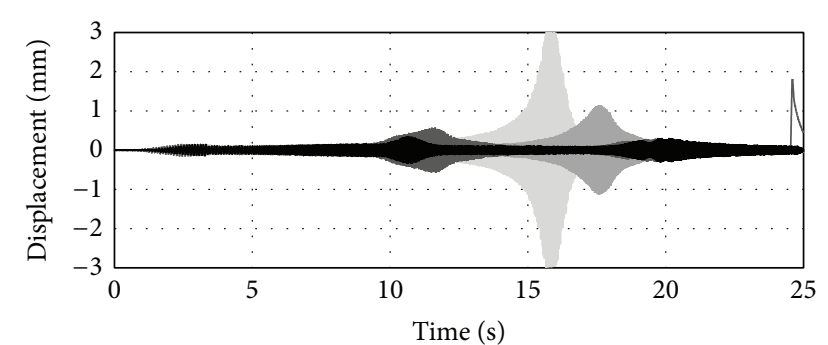

$\begin{array}{ll}\text { Without damper } & -4 \mathrm{~A} \\ 0 \mathrm{~A} & - \text { Variable }\end{array}$

FIGURE 13: Time histories of the primary system response excited by swept-sine input at the speed of $2 \mathrm{~Hz} / \mathrm{sec}$.

obtained when an electric current of $4 \mathrm{~A}$ is applied. If we assume use of a damper with an optimal damping property, the amplitudes of the two distinct resonance peaks may decrease to the level of the two fixed points, while the amplitude within the frequency range between the points will increase, as predicted by the numerical investigation. On the other hand, outside the range, the amplitude will stay at the same level as the case where only the optimal tuning is considered. Consequently, the overall response of the adaptively tuned absorber is thought to outperform the response of the optimally tuned and damped absorber.

These results demonstrate that the frequency-tunable dynamic absorber using MRE works effectively for the vibration control of structures, while keeping the mechanism simple.

\section{Conclusions}

In this study, the dynamic property of the stiffness controllable elastomer, known as MRE, is experimentally evaluated. The dynamic property test shows that a certain optimal composition of materials within the mixture, in this case a 40 vol\% iron content, leads to a maximum stiffness property change. On the other hand, the loss factor is found to be almost constant irrespective of the presence of the magnetic field. The MRE is then used in a DVA in order to provide natural frequency tunability. The absorber is further applied to the vibration control of a one-DOF structure in conjunction with a real-time stiffness switching algorithm, where the damping performance of the absorber is evaluated numerically and experimentally. To attain the effective design of the adaptive dynamic absorber and maximize the damping performance, the natural frequency of a target structure must be located at the middle of the variable frequency range of the absorber. Moreover, the swept-sine excitation results of a target structure demonstrate that better damping performance can be obtained by the real-time frequency-tunable dynamic absorbers than by passive-type absorbers. The proposed tunable dynamic absorber using MREs therefore enhances the damping performance with simple alteration of the stiffness element in the conventional passive-type absorber that works effectively for the vibration control of structures.

\section{Conflict of Interests}

The authors declare that there is no conflict of interests regarding the publication of this paper.

\section{Acknowledgment}

The work was financially supported by the Japan Ministry of Education, Culture, Sports, Science and Technology, Grantin-Aid for Research (J-KAKEN), Category (C), no. 24560256.

\section{References}

[1] J. P. den Hartog, Mechanical Vibrations, McGraw-Hill, 1956.

[2] J. Q. Sun, M. R. Jolly, and M. A. Norris, "Passive, adaptive and active tuned vibration absorbers-a survey," Journal of Vibration and Acoustics, vol. 117, pp. 234-242, 1995.

[3] K. Nagaya, A. Kurusu, S. Ikai, and Y. Shitani, "Vibration control of a structure by using a tunable absorber and an optimal vibration absorber under auto-tuning control," Journal of Sound and Vibration, vol. 228, no. 4, pp. 773-792, 1999.

[4] P. L. Walsh and J. S. Lamancusa, "A variable stiffness vibration absorber for minimization of transient vibrations," Journal of Sound and Vibration, vol. 158, no. 2, pp. 195-211, 1992.

[5] S. Nagarajaiah and E. Sonmez, "Structures with semiactive variable stiffness single/multiple tuned mass dampers," Journal of Structural Engineering, vol. 133, no. 1, pp. 67-77, 2007.

[6] T. Komatsuzaki, Y. Iwata, and S. Morishita, "Semi-active vibration control of structures using MR elastomers," in Proceedings of the 14th Asia-Pacific Vibration Conference (APVC '11), S. S. Law, L. Cheng, Y. Xia, and Z. Su, Eds., vol. 4, pp. 1875-1884, Hong Kong, China, 2011.

[7] G. J. Liao, X.-L. Gong, S. H. Xuan, C. J. Kang, and L. H. Zong, "Development of a real-time tunable stiffness and damping vibration isolator based on magnetorheological elastomer," Journal of Intelligent Material Systems and Structures, vol. 23, no. 1, pp. 25-33, 2012.

[8] H. X. Deng and X. L. Gong, "Adaptive tuned vibration absorber based on magnetorheological elastomer," Journal of Intelligent Material Systems and Structures, vol. 18, no. 12, pp. 1205-1210, 2007.

[9] A. A. Lerner and K. A. Cunefare, "Performance of MRE-based vibration absorbers," Journal of Intelligent Material Systems and Structures, vol. 19, no. 5, pp. 551-563, 2008.

[10] N. Hoang, N. Zhang, and H. Du, "An adaptive tunable vibration absorber using a new magnetorheological elastomer for vehicular powertrain transient vibration reduction," Smart Materials and Structures, vol. 20, no. 1, Article ID 015019, 2011.

[11] A. V. Chertovich, G. V. Stepanov, E. Y. Kramarenko, and A. R. Khokhlov, "New composite elastomers with giant magnetic response," Macromolecular Materials and Engineering, vol. 295, no. 4, pp. 336-341, 2010.

[12] X. L. Gong, X. Z. Zhang, and P. Q. Zhang, "Fabrication and characterization of isotropic magnetorheological elastomers," Polymer Testing, vol. 24, no. 5, pp. 669-676, 2005.

[13] X. Zhang, S. Peng, W. Wen, and W. Li, "Analysis and fabrication of patterned magnetorheological elastomers," Smart Materials and Structures, vol. 17, no. 4, Article ID 045001, pp. 1-5, 2008.

[14] M. R. Jolly, J. D. Carlson, B. C. Muñoz, and T. A. Bullions, "The magnetoviscoelastic response of elastomer composites 
consisting of ferrous particles embedded in a polymer matrix," Journal of Intelligent Material Systems and Structures, vol. 7, no. 6, pp. 613-622, 1996.

[15] L. C. Davis, "Model of magnetorheological elastomers," Journal of Applied Physics, vol. 85, no. 6, pp. 3348-3351, 1999.

[16] G. Y. Zhou, "Shear properties of a magnetorheological elastomer," Smart Materials and Structures, vol. 12, no. 1, pp. 139146, 2003.

[17] S. J. Dyke, B. F. Spencer Jr., M. K. Sain, and J. D. Carlson, "Modeling and control of magnetorheological dampers for seismic response reduction," Smart Materials and Structures, vol. 5, no. 5, pp. 565-575, 1996.

[18] J. D. Carlson and M. R. Jolly, "MR fluid, foam and elastomer devices," Mechatronics, vol. 10, no. 4, pp. 555-569, 2000.

[19] N. Shimizu and H. Yamazaki, "Development of vibration insulator using a new material, silicone gel," Transactions of the Japan Society of Mechanical Engineers, Series C, vol. 59, no. 568, pp. 3717-3724, 1993. 

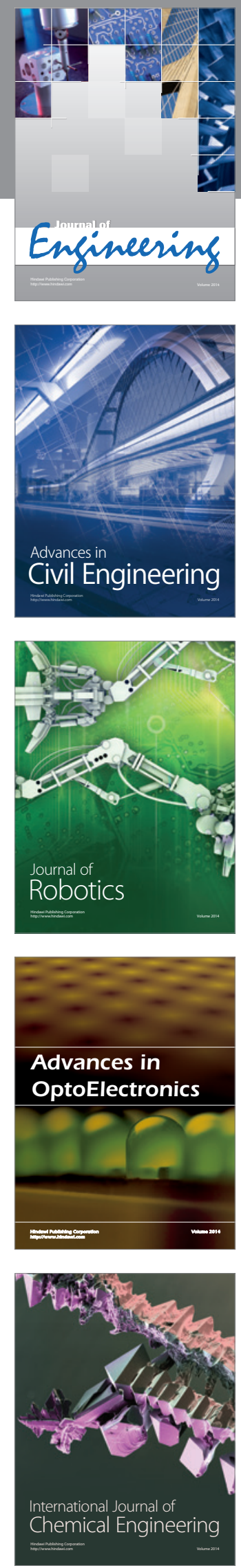

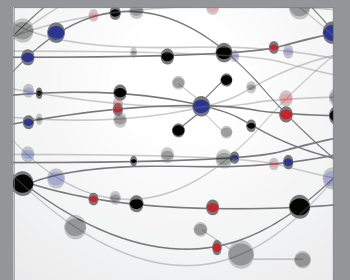

The Scientific World Journal
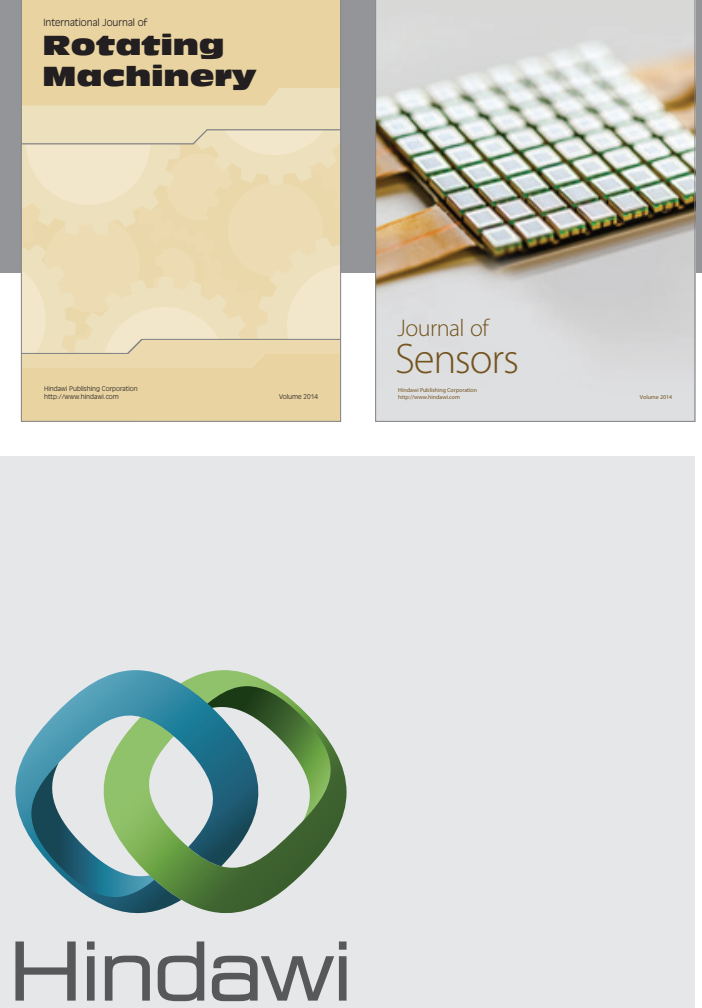

Submit your manuscripts at http://www.hindawi.com
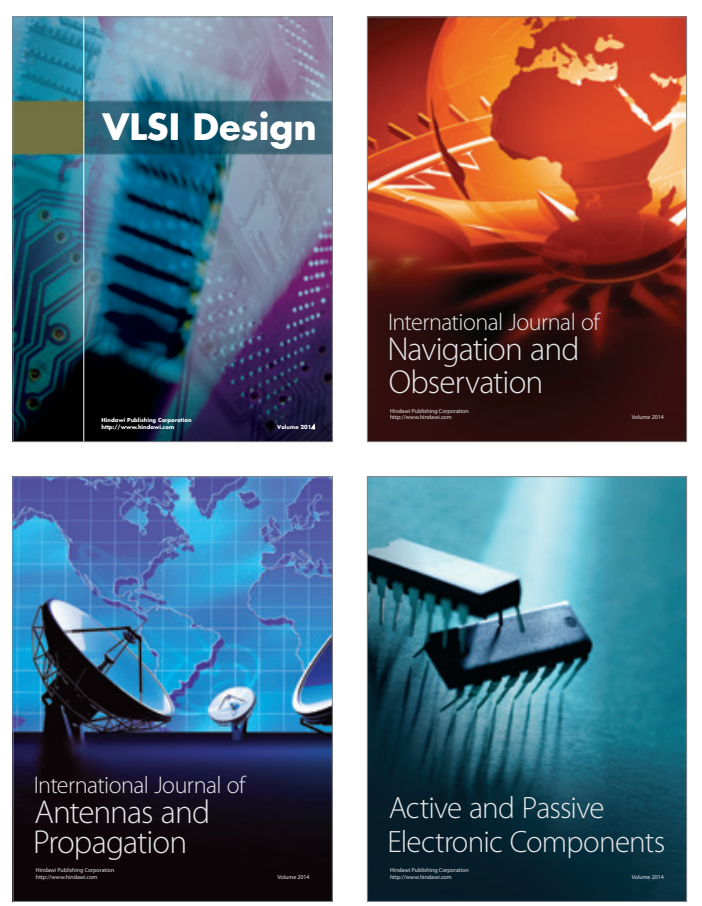
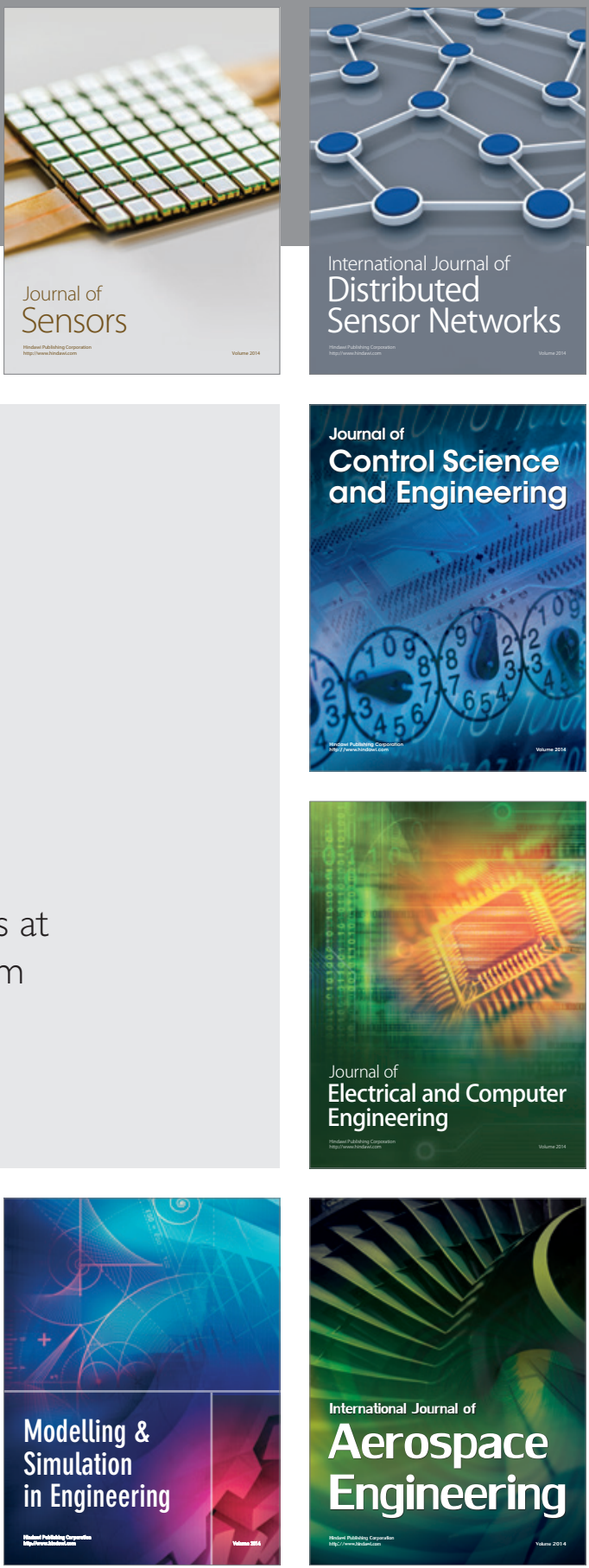

Journal of

Control Science

and Engineering
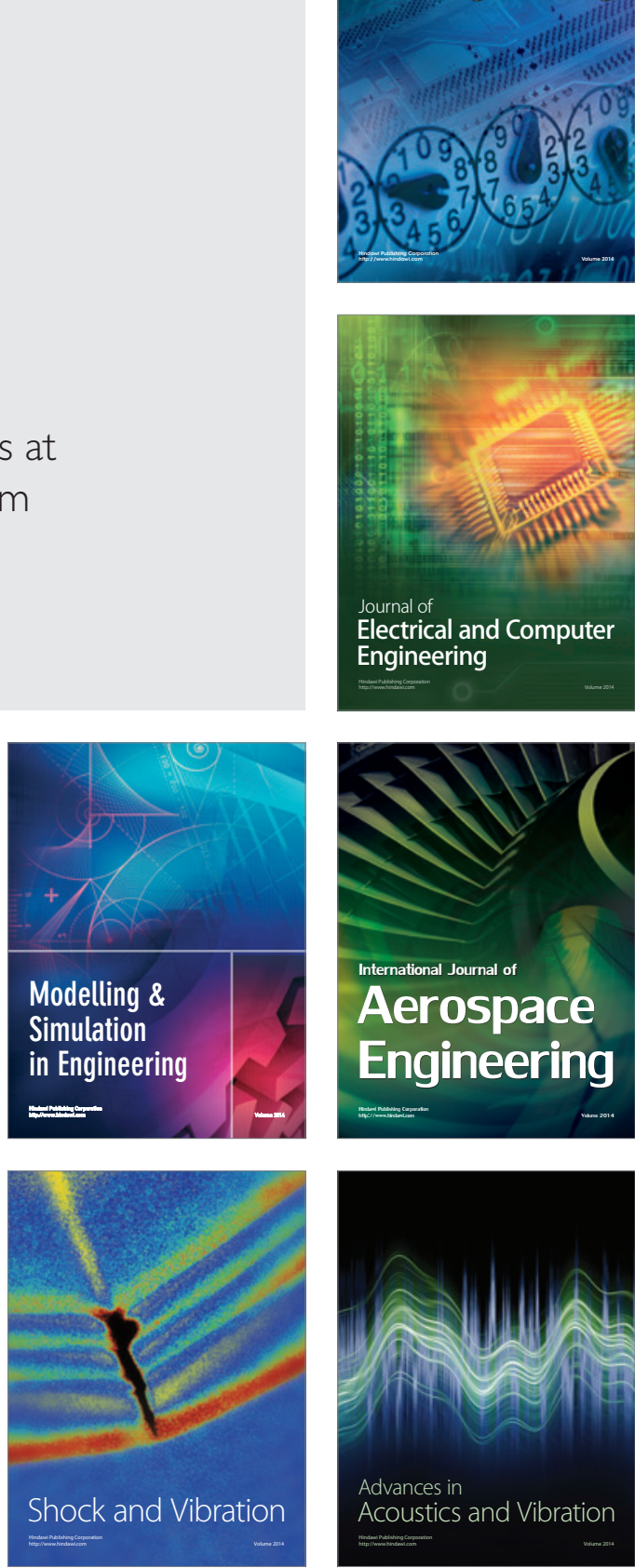